\title{
PERCEPCIÓN DE LA CALIDAD DE SERVICIO DE LOS USUARIOS EN EL CONSULTORIO EXTERNO DE MEDICINA INTERNA DEL HOSPITAL AUGUSTO HERNÁNDEZ MENDOZA ESSALUD - ICA EN FEBRERO-MARZO DEL 2017 Perception Of Service Quality Of Users In The External Office Of Internal Medicine Of Hospital Augusto Hernández MendozaEssalud - Ica In February-march Of 2017
}

\section{ARTÍCULO ORIGINAL}

Joel Custodio-Velásquez ${ }^{1, a}$, Claudia del rosario Roja-Valdez ${ }^{2, a}$, Luis Hernández-Cabrera ${ }^{3, a}$

1. Dirección Regional de Salud Ica. Ica, Perú.

2. Organización de Centros de Prestación del Servicio del Asegurado Es salud -Ica.

3. Hospital San Juan de Dios de Pisco. Ica, Perú.

a. Médico Cirujano

Correspondencia:

Joel Custodio Velásquez

Domicilio: Urb San Martin de Porres

D 18 - Ica

Telefono: 942277343

Correo Electrónico:

Joel_acuario_212303@hotmail.com

Contribuciones De Autoría:

CVJ, RVCR, HCL participaron en el diseño del estudio, el análisis de los datos, revisaron críticamente el artículo y aprobaron la versión final.

Conflicto De Intereses: No declarados.

Financiamiento: Autofinanciado.

Recibido: 05-11-2017

Aceptado: 20-11-2017

Publicado: 20-12-2017

\section{Como Citar}

Custodio Velásquez J, Rojas Valdez Claudia del rosario, Hernández Cabrera L. Percepción de la calidad de servicio de los usuarios en el consultorio externo de medicina interna del hospital augusto Hernández Mendoza - Es salud - Ica en febrero-marzo del 2017. Rev méd panacea. 2017;6 (3) $111-115$

\begin{abstract}
RESUMEN:
Objetivo: Describir la percepción de la calidad de servicio de los usuarios en el consultorio externo de medicina interna del Hospital Augusto Hernández Mendoza - Essalud - Ica en febrero-marzo del 2017. Diseño de Estudio: Descriptivo de tipo transversal. Nivel de investigación Observacional. Material y Metodos: Se seleccionó de manera no probabilística una muestra de 130 usuarios de los consultorios externos del Hospital Augusto Hernández Mendoza - Essalud - Ica en febrero-marzo del 2017. Se utilizó muestreo no probabilístico, empleándose la encuesta SERVQUAL modificada a 22 pares de preguntas el cual mide la satisfacción calculando la diferencia entre las respuestas para las expectativas y las percepciones. Los datos fueron analizados con el paquete estadístico SPSS 24.0. Resultados: Se halló una insatisfacción global de $60.8 \%$ e insatisfacción en las dimensiones de respuesta rápida de $62.3 \%$; las dimensiones de: confiabilidad (50.8\%), Aspectos Tangibles (56.2\%), empatía (48.5\%) y seguridad (46.9\%). Conclusiones: La insatisfacción encontrada (60.8\%) es semejante a la realizada en servicios de Essalud y relativamente más baja que la realizada en servicios de salud de MINSA. Las expectativas son altas en comparación a las percepciones. No se encontró asociación estadísticamente significativa entre los factores sociodemográficos y la satisfacción global ni las dimensiones.
\end{abstract}

Palabras Claves: Encuesta SERVQUAL, nivel de satisfacción, calidad de servicio.

\begin{abstract}
:
Objetive: To describe the user perception of quality in the internal medicine outpatient service at August Hernandez Mendoza Hospital in february - march 2017. Design: Descriptive, Cross-sectional and Observational study. Material And Methods: patients $(n=130)$ was selected in a non -probabilistic way using the modified SERVQUAL scale (22 pairs of questions) wich measures the level of satisfaction by calculating the difference between the responses to the expectations and perceptions. Data were analyzed with SPSS 24.0. Results: we found a global dissatisfaction of $83.9 \%$ and dissatisfaction in rapid response's $62.3 \%$; dimensión: reliability (50.8\%), the tangible dimensions (56.2\%), safety (46.9\%). and empathy (48.5\%) Conclusions: The dissatisfaction is higher in comparisson to other studies. The expectations higher tan perceptions. There's no statistically significant association between sociodemographic factors and global satisfaction neither about dimensions.
\end{abstract}

Keywords: SERVQUAL scale, level of satisfacction, service's quality. 


\section{INTRODUCCIÓN}

En nuestro medio a partir de los años 90, se inician estudios sobre calidad de servicio pero de salud reproductiva. En 1992, el informe de la Encuesta de Disponibilidad de Servicios de Planificación Familiar (EDIS-PERU 1992), donde señala de un 80 por ciento de satisfacción del usuario.

Aphang(1) en 1997 realizó un estudio en los mismos consultorios externos, mediante una encuesta no validada previamente $\mathrm{y}$ obtuvo como resultado que el $98 \%$ de los pacientes entrevistados estuvo satisfecho con el servicio recibido. Seclén-Palain(2), en el análisis de datos secundarios de la Encuesta Nacional de Vida realizada en el año 2000 , encontró $62,1 \%$ de satisfacción del usuario en hospitales. Acosta (3), en el 2002 reportó un 96\% de satisfacción en las expectativas de los usuarios de los consultorios externos de medicina del HNAL con insatisfacción de 70,33\% para el tiempo de espera. Seclén-Palain (2) abordan la satisfacción del usuario como 'enfoque de resultado', midiendo directamente la satisfacción global con la atención más que como el 'enfoque de proceso' que pone atención en los componentes de la atención y es el enfoque que se sigue mediante el uso del instrumento SERVQUAL.

Núñez (4) en este mismo servicio de Medicina Interna, con la metodología SERVQUAL mostró niveles de insatisfacción leve a moderada en $53 \%$ y severa en $2 \%$. Casalino-Carpio (5) también en los consultorios externos del HNAL encontró una insatisfacción leve a moderada de $54,03 \%$ e insatisfacción severa de 1,61\%. Como comparación en Lima, en la consulta externa del servicio de Medicina Interna del Hospital Nacional Hipólito Unanue, en 2005, se encontró, una insatisfacción leve a moderada de 94,4\% e insatisfacción severa en 1,7\%.(6). Cabello y Chirinos (7) aplicando la encuesta SERVQUAL obtuvo como resultado insatisfacción del 52.9\% en el HNAL en el 2011. Rodriguez y Caballero(8) utilizando una encuesta validada distinta al SERVQUAL obtuvieron como resultado insatisfacción en los diversos ámbitos hospitalarios entre el $54 \%$ al $90 \%$ en el centro de diagnóstico terapéutico del hospital San Juan de Dios 2013. Nimango (9) en el consultorio externo de medicina interna del Hospital Nacional Arzobispo Loayza en enero del 2014 obtuvo como resultados la insatisfacción encontrada 83,9\%. De la Cruz(10) en los consultorios externos de Medicina Interna del Hospital Nacional Dos de Mayo en Diciembre del 2015 halló una insatisfacción global de $55.7 \%$ e insatisfacción en las dimensiones de capacidad de respuesta de $91.5 \%$; seguida de las dimensiones de fiabilidad (70.4\%), Aspectos Tangibles (42.2\%), empatía (39.1\%) y seguridad (35.8\%). Villar (11) en los servicios de medicina complementaria del Seguro Social de Salud ubicados en Lima y Callao durante enero 2016 obtiene como resultado una satisfacción promedio de $68.9 \%$. En provincia: Rey Luces (12) en el Hospital Santa Margarita de Andahuaylas el 2015 obtuvo que $50.19 \%$ tienen una percepción de la calidad de atención como Regular, un 37,55\% perciben la calidad de atención como Mala y solo un $12,27 \%$ tienen una percepción Buena.

La justificación del estudio es que la evaluación de la calidad de servicio desde la percepción del usuario externo, cada vez, es más común, a partir de ello, es posible obtener del usuario un conjunto de conceptos y actitudes en relación con el servicio recibido, los cuales beneficiará a la organización que brinda los servicios de salud y al usuario satisfaciendo sus necesidades y expectativas (13). Se planteó como objetivo Evaluar la calidad de servicio en consulta externa del Hospital Augusto Hernández Mendoza - Essalud - Ica en febrero-marzo del 2017 e Identificar el nivel de expectativas y percepciones acerca de la calidad en el servicio de consulta externa en el Hospital Augusto Hernández Mendoza - Essalud - Ica en febrero-marzo del 2017; comparar el nivel de expectativas según el nivel de percepción sobre la calidad en el servicio de consulta externa y distinguir las características más importantes que las personas consideran en el servicio de consulta externa del Hospital Augusto Hernández Mendoza - Essalud - Ica en febrero-marzo del 2017.

\section{MATERIAL Y MÉTODOS}

Se plantea un diseño de estudio Descriptivo de tipo transversal. Nivel de investigación Observacional. Población :Usuarios de consulta externa de medicina interna del Hospital Augusto Hernández Mendoza Essalud Ica en el mes de febrero- marzo del 2017.Unidad de análisis :Usuario de consulta externa de medicina interna del Hospital Augusto Hernández Mendoza Essalud Ica en el mes de febrero- marzo del 2017.Tipo de muestra y tamaño: No Probabilístico - Intencional

La muestra está formada por aquellos usuarios que asistieron al consultorio externo de medicina interna del HAHM durante la última semana del mes de febrero y primera semana de marzo del 2017 que cumplieron con los criterios de inclusión y exclusión determinados en la investigación. Si la población es finita, es decir conocemos el total de la población y deseásemos saber cuántos del total tendremos que estudiar la fórmula es: $\mathrm{n}=\mathrm{Z} 2 \mathrm{pqN} / \mathrm{e2}(\mathrm{N}-1)+\mathrm{z} 2 \mathrm{pq}$. Dónde: n: Tamaño de muestra .p: Proporción de usuarios externos que espera que se encuentren insatisfechos, $p=0.5$.q: Proporción de usuarios externos que espera que se encuentren satisfechos. Su valor es (1-p), q = 0.5.e: Error Estándar de 0.05 ó o.1 según categoría del establecimiento, e=0.05 .z: Valor de "z" para el intervalo de confianza 95\%. El valor de "z" es igual a 1.96.N: Población de usuarios externos atendidos durante las fechas recomendadas en los servicios de consulta externa.

\section{Criterios de inclusión y exclusión:}

Criterios de Inclusión: Usuarios de consultorios externos de medicina interna del HAHM ambos sexos. Usuarios $\geq$ de 18 años. Usuarios sin ninguna alteración de la conciencia y capaces de comunicarse en español. Aceptación voluntaria de participar en el estudio luego de haber sido informados verbalmente de las características y objetivos de este, firmando consentimiento informado. Usuarios nuevos o continuadores en el servicio.

Criterios de Exclusión: Usuarios que presenten algún tipo de discapacidad o trastorno mental por la que no puedan expresar su opinión y que no estén con familiares o acompañante alguno. Encuestas llenadas de forma equivocada o incompleta. Usuarios que no acepten firmar el consentimiento informado.

Técnica e instrumento de recolección de datos: Se tomó como fuente de información para medir el nivel de satisfacción del usuario según la calidad de servicio del consultorio externo de medicina interna del HAHM, la realización de la encuesta SERVQUAL modificada para establecimientos de salud. El cuestionario tipo SERVQUAL fue desarrollado por Parazuraman, A. Berry L. y Zeithalm para medir satisfacción del usuario en empresas de servicios, luego adaptada y validada por Barbakus, Maugold para ser aplicada en hospitales.

El mismo cuestionario fue validado también en nuestro medio, Emilio Cabello (14). El SERVQUAL mide la calidad de servicio a través de la encuesta que consta de cuatro áreas: $1^{\circ}$ Datos generales del encuestado. $2^{\circ}$ Preguntas de las expectativas del usuario, sobre el servicio que esperaría por recibir, en los consultorios externos del HAHM. $3^{\circ}$ Preguntas sobre la percepción de la calidad de servicio recibido. $4^{\circ}$ Cuáles son las priorizaciones de la dimensión de la calidad. Las encuestas para el servicio de Consulta Externa incluyen en su estructura 22 preguntas de Expectativas y 22 preguntas de Percepciones, distribuidas en cinco criterios o dimensiones de evaluación de la calidad: Fiabilidad: Preguntas del 01 al 05.Capacidad de Respuesta: Preguntas del 06 al 09.Seguridad: Preguntas del 10 al 13 .Empatía: Preguntas del 14 al 18 Aspectos Tangibles: Preguntas del 19 al 22.

Las áreas de preguntas (Expectativas y Percepción) están diseñadas al tipo de escala de Likert del 1 al 7, considerando 1 la más baja y 7 , la más alta.

El grado de satisfacción de los usuarios se determinó calculando la diferencia entre las respuestas brindadas para las expectativas y las percepciones; posteriormente fue interpretada según los siguientes parámetros: 1 . Menor de $0=$ satisfacción amplia. 2. $0=$ 
satisfacción. 3. Mayor que o y menor o igual a 2 = insatisfacción leve/moderada. 4. Mayor que 2 = insatisfacción severa.

La encuesta fue distribuida por personal ajeno al servicio, previamente capacitado, a usuarios de los consultorios externos del HAHM ubicados en el mono block, se explicó a los pacientes detalladamente sobre la forma adecuada del llenado de la encuesta, la cual tuvo una duración por cada usuario entre 10 a 15 min, la encuesta constó de dos etapas: la primera etapa consistió en contestar las 22 preguntas sobre las expectativas, antes de recibir el servicio y la segunda etapa en contestar las 22 preguntas sobre la percepción, después de haber recibido el servicio.

Procesamiento de datos: Los resultados fueron tabulados en Excel 2013 y después fueron trabajados por el paquete estadístico SPSS 24.0, se realizó el análisis descriptivo de todas las variables, a través de la determinación de proporciones de las variables sociodemográficas y medias de satisfacción de expectativas y percepciones; el cálculo de las brechas entre expectativas y percepciones por ítems y dimensiones; el grado de satisfacción de los usuarios según los parámetros anteriormente mencionados; el análisis bivariado de las medias de satisfacción global y de cada una de las dimensiones de la calidad de servicio según variables sociodemográficas, se buscaron asociación empleando el Chi2 con un nivel de confianza de $95 \%$, la identificación de los factores relacionados a la satisfacción global y la satisfacción de cada una de las dimensiones de la calidad se utilizó el análisis de correlación de Pearson, para las variables cuantitativas, el test T de Student para la asociación entre los puntajes de las dimensiones expectativas y percepciones globales

\section{RESULTADOS}

Se encuestaron 130 personas, donde se encontró una media de 56 años, con una mínima edad de 18 años y una máxima edad de 89 años. (Figura 1)

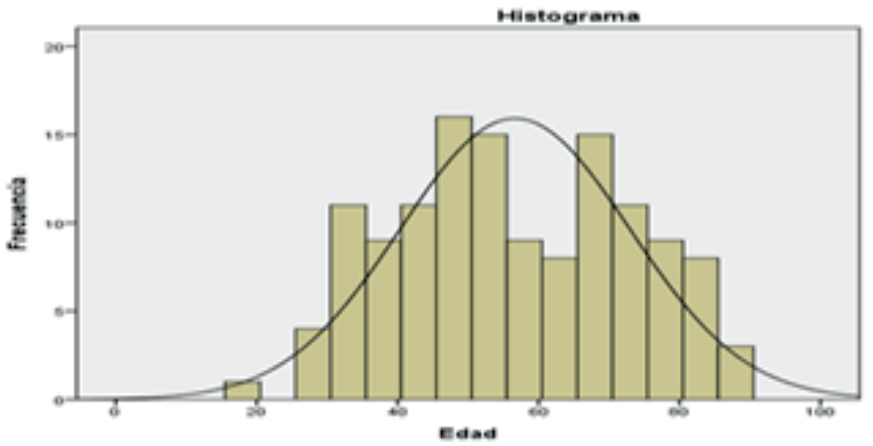

Media $=56.65 / /$ Desviación estándar $=16,298 / / N=130$

Figura 1: Histograma

Tabla 1: Caracteristicas de los usuarios

\begin{tabular}{|c||c|c|}
\hline & Frecuencia & $\%$ \\
\hline Edad & 56.65 & DS (16.298) \\
Género & & \\
Masculino & 54 & $41.50 \%$ \\
Femenino & 76 & $58.50 \%$ \\
Nivel de Estúdio & & \\
Analfabeto & 2 & $1.50 \%$ \\
Primaria & 27 & $20.80 \%$ \\
Secundaria & 34 & $26.20 \%$ \\
Superior Técnico & 9 & $6.90 \%$ \\
Superior Universitario & 58 & $44.60 \%$ \\
Tipo de Usuario & & \\
Nuevo & 32 & $24.60 \%$ \\
Continuador & 98 & $75.40 \%$ \\
\hline
\end{tabular}

El nivel de instrucción que muestran los encuestados, 1.54\% (02) son analfabetos, el $20.77 \%$ (27 participantes) tienen nivel primario, $26.15 \%$ (34) nivel secundario, $6.92 \%$ (09) tienen superior técnico y el $44.62 \%$ (58) tienen superior universitario, el $75.4 \%$ (98) era usuario continuador y el $24.6 \%$ (32) era usuario nuevo.

\section{Grado de Satisfacción del Usuario}

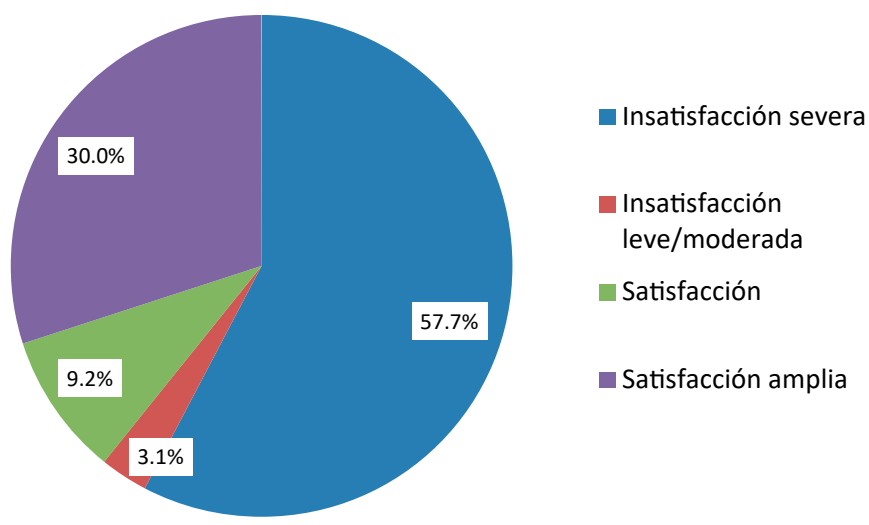

Figura 2: Grado de satiscfacción

El grado de satisfacción que presentan los usuarios, el 30\% (39) presentan satisfacción amplia, el 9.2\% (12) satisfacción, el 3.1\% (04) una insatisfacción leve a moderada y el $57.7 \%$ (75) una insatisfacción severa, siendo así que el 60\% presenta insatisfacción de la atención médica por consultorio externo (Figura 2)

\begin{tabular}{|l|c|c|c|c|}
\hline \multicolumn{5}{|c|}{ Grado de Satisfacción } \\
\hline Aspectos tangibles & 36.2 & 20 & 18.6 & 25.4 \\
\hline Usuario empatia & 30 & 18.5 & 26.2 & 25.4 \\
\hline \begin{tabular}{l|l|l|l|} 
Usuario seguridad \\
Usuario capacidad
\end{tabular} & 33.1 & 13.8 & 25.4 & 27.7 \\
\hline \begin{tabular}{l} 
Usuario fiabilidad \\
\hline
\end{tabular} & 42.3 & 20 & 16.9 & 20.8 \\
\hline & 37.7 & 13.1 & 18.5 & 30.8 \\
\hline & Satisfacción & & & $\begin{array}{l}\text { Insatisfacción leve/moderada } \\
\text { Satisfacción amplia }\end{array}$ \\
\hline
\end{tabular}

Figura 3: Grado de satisfacción de usuario

En la evaluación por dimensión de la evaluación de calidad, los usuarios presentan una insatisfacción severa en la capacidad de respuesta, en la fiabilidad y en aspectos tangibles, con más del 50\%, por el contrario los que presentan más del $50 \%$ de satisfacción, son en las dimensiones de seguridad y de empatía (Figura 3).

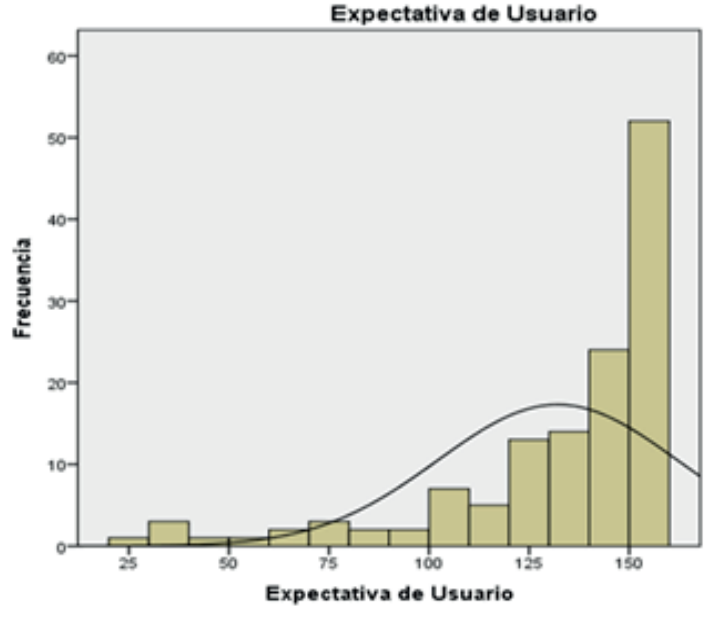

Media = 132.14 // Desviación estándar = 29,955 // N=130 


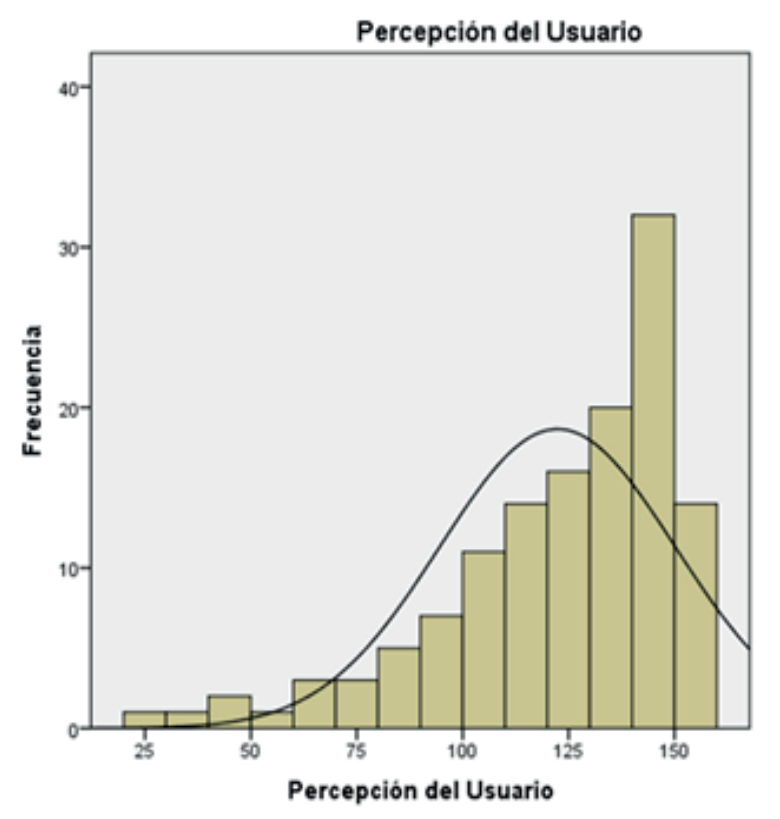

Media $=122.38$ // Desviación estándar $=27,799 / / \mathrm{N}=130$

Figura 5: Percepción del usuario

Respecto a los puntajes obtenidos en el caso de las expectativas, los usuarios de los servicios de la consulta externa del HAHM son altas, pero que después de recibir el servicio, las percepciones de los usuarios cambiaron hacia un puntaje menor al inicial (Figura $4 \mathrm{y}$ 5). Existe una diferencia significativa (t-student, sig.: 0.000) (Tabla $\mathrm{N}^{\circ}$ )

En la correlación por los puntajes obtenidos entre la expectativa y percepción que tienen los usuarios del servicio médico, podemos apreciar en la Figura 7, que si bien existe una tendencia lineal positiva, el grado de porcentaje de la variabilidad de los datos que explica la asociación entre las dos variables es bajo $(R 2=0.34)$, mostrándose en el grafico que se obtiene percepción baja del servicio cuando se tiene una expectativa alta.

\section{DISCUSIÓN}

En nuestro proyecto se encuentra una insatisfacción de $60.77 \%$ semejante a la encontrada en otros trabajos realizados en EsSalud el 2015 con insatisfacción del 56\%(11) y menor que los encontrados en trabajos realizados en el sector MINSA donde se encuentran porcentajes de insatifacción mayores al $70 \%(4,5))$ llegando inclusive en algunas dimensiones a mayor de $80 \%(8,9)$

El presente trabajo arroja que la dimensión con mayor nivel de insatisfacción expectativa-percepción es capacidad de respuesta insatisfacción (62.3\%), lo cual coincide con todos los resultados de los trabajos realizados en los últimos 10 años ya sea en entidades de MINSA o EsSalud, siendo las otras dimensiones las que son cambiantes en cuanto a su representatividad en los diversas entidades $(7,9)$

Las dimensiones con menor nivel de insatisfacción son empatía (48.5\%) y seguridad (36.9\%) resultados distintos a los descritos en otros trabajos donde ambas son mayores al $50 \%$ ya sea en entidad de MINSA, EsSalud o Fuerzas policiales $(6,8)$

No se encontró relación entre la satisfacción global y los factores sociodemográficos estudiados (sexo, grado de instrucción y de usuario), respecto a la calidad de servicio que se oferta en los consultorios externos del servicio de Medicina Interna del Hospital Augusto Hernández Mendoza - Essalud - Ica en febrero-marzo del 2017, lo que indica que no es relevante el estrato social o nivel intelectual. 


\section{BIBLIOGRAFÍA}

1. Aphang M, Casas J. Calidad de la atención en la consulta ambulatoria de Medicina Interna en el Hospital Arzobispo Loayza. HAL Revista Científica del Cuerpo Médico del Hospital Arzobispo Loayza 1977;2:24-8.

2. Seclén-Palacin J, Darras C. Satisfacción de usuarios de los servicios de salud: Factores sociodemográficos y de accesibilidad asociados. Perú, 2000. An Fac Med Lima 2005;66:127-41.

3. Acosta Z. Percepción de la calidad de atención en los consultorios externos de medicina desde la perspectiva de la satisfacción de los usuarios del Hospital Nacional Arzobispo Loayza, 2002. [Tesis de Maestría] Universidad Peruana Cayetano Heredia, Lima, 2002.

4. Núñez Z. Estudio de evaluación de la calidad de servicio de los consultorios externos del servicio de medicina del HNAL Lima 2006. [Tesis de Maestría] Universidad Peruana Cayetano Heredia, Lima, 2006.

5. Casalino-Carpio. Calidad de servicio de la consulta externa de Medicina Interna de un hospital general de Lima mediante la encuesta Servqual. Rev Soc Peru Med Interna 2008; vol 21 (4). Lima.Perú.2008.

6. Ricci V. Calidad de servicio percibida por los usuarios de la consulta externa de Medicina Interna del Hospital Nacional Hipólito Unanue, 2005. [Tesis de Maestría] Universidad Peruana Cayetano Heredia, Lima, 2007.

7. Cabello E, Chirinos J. Validación y aplicabilidad de encuestas SERVQUAL modificadas para medir la satisfacción de usuarios externos en servicios de salud. Rev Med Hered. 2012; 23:88-95 8. Rodriguez N, Caballero K. Calidad de atención, desde la perspectiva del usuario en el centro de diagnóstico terapéutico del Hospital San Juan de Dios [tesis para optar el título de administrador público y gestión púbica] Chile: Escuela de posgrado. Universidad Academia de Humanismo Cristiano 2013.

9. Ninamango W. Percepción de la calidad de servicio de los usuarios en el consultorio externo de medicina interna del Hospital Nacional Arzobispo Loayza en Enero del 2014[tesis doctoral] Facultad de medicina. E.A.P. de medicina humana. Lima - Perú 2014 10. De la Cruz J. Nivel de satisfacción de los usuarios en consultorios externos de Medicina Interna del Hospital Nacional Dos de Mayo en Diciembre del 2015.[tesis Doctoral] Lima escuela de postgrado Universidad Nacional Mayor de San Marcos 2015.

11. Villar M. Determinar la percepción de la calidad de los servicios de medicina complementaria del Seguro Social de Salud ubicados en Lima y Callao. Revista Peruana de Medicina Integrativa.2016; 1(3):27-33.

12. Huarcaya R. Percepciòn de la calidad de atención en los usuarios del servicio de emergencia del hospital I Santa Margarita de Andahuaylas 2015 [Tesis para Oaptar el Título Profesional de Licenciado en gerencia en salud] Andahuaylas .Escuela profesional de Administraciòn de Empresas. Universidad Nacional Josè Marìa Arguedas 2015

13. Delgado M, Vázquez M., Moraes L. Calidad en los servicios de salud desde los marcos de sentido de diferentes actores sociales en Colombia y Brasil. Rev. salud pública 2010; 12(4): 533-545.

14. Cabello E, Chirinos J. Validación y aplicabilidad de encuestas SERVQUAL modificadas para medir la satisfacción de usuarios externos en servicios de salud. Rev Med Hered. 2012; 23:88-95 